\title{
NANOGP8 expression regulates gastric cancer cell progression by transactivating DBC1 in gastric cancer $\mathrm{MKN}-45$ cells
}

\author{
LI LI $^{1,2^{*}}$, RU FENG ${ }^{3 *}$, SUJUAN FEI ${ }^{2}$, JIANG CAO ${ }^{4}$, QINQIN ZHU ${ }^{2}$, GUOZHONG JI $^{5}$ and JIANWEI ZHOU ${ }^{1}$ \\ ${ }^{1}$ Department of Molecular Cell Biology and Toxicology, Jiangsu Key Laboratory of Cancer Biomarkers, \\ Prevention and Treatment, Cancer Center, Nanjing Medical University, Nanjing, Jiangsu 211166; \\ ${ }^{2}$ Department of Gastroenterology, The Affiliated Hospital of Xuzhou Medical University, Xuzhou, Jiangsu 221000; \\ ${ }^{3}$ Department of Geriatrics, Suqian People's Hospital Drum Tower Hospital Group, Suqian, Jiangsu 223800; \\ ${ }^{4}$ Department of Hematology, The Affiliated Hospital of Xuzhou Medical University, Xuzhou, Jiangsu 221000; \\ ${ }^{5}$ Department of Medical Examination Center and Institute of Digestive Endoscopy and Medical Center for Digestive Diseases, \\ The Second Affiliated Hospital of Nanjing Medical University, Nanjing, Jiangsu 210000, P.R. China
}

Received February 25, 2017; Accepted July 23, 2018

DOI: $10.3892 / \mathrm{ol} .2018 .9595$

\begin{abstract}
NANOGP8 is one of the NANOG pseudogenes and is expressed together with NANOG in multiple tumor tissues and cell lines. The biological functions of NANOGP8 in progression of gastric cancer are unclear. In the present study, the role of NANOGP8 was investigated in gastric cancer cells. The gathered data demonstrated that NANOG expression in both mRNA and protein was elevated in gastric cancer cell lines relative to a normal gastric epithelial cell line. Downregulation of NANOGP8 inhibited cell proliferation and increased apoptosis in human gastric carcinoma cell lines. Furthermore, silencing of NANOGP8 suppressed tumor growth in vivo. Interestingly, it was identified that deleted in breast cancer 1 (DBC1) expression was also markedly downregulated following NANOGP8 knockdown. DNA microarray and dual-luciferase assays further indicated that NANOGP8 may bind to the $\mathrm{DBC} 1$ promoter region and regulate $\mathrm{DBC} 1$ expression. Therefore, the gathered data provided evidence that NANOGP8 contributes to progression of gastric cancer via $\mathrm{DBCl}$ and may have potential translational significance.
\end{abstract}

Correspondence to: Professor Jianwei Zhou, Department of Molecular Cell Biology and Toxicology, Jiangsu Key Laboratory of Cancer Biomarkers, Prevention and Treatment, Cancer Center, Nanjing Medical University, 101 Longmian Avenue, Nanjing, Jiangsu 211166, P.R. China

E-mail: jwzhou@njmu.edu.cn

Professor Guozhong Ji, Department of Medical Examination Center and Institute of Digestive Endoscopy and Medical Center for Digestive Diseases, The Second Affiliated Hospital of Nanjing Medical University, 121 Jiangjiayuan, Nanjing, Jiangsu 210000, P.R. China E-mail: jgzzl@163.com

*Contributed equally

Key words: proliferation, apoptosis, gastric cancer

\section{Introduction}

Gastric cancer is one of the most common diseases and the third leading cause for cancer-associated mortality in the world (1). It was reported that in 2010 gastric cancer cases in China lead to a mortality rate of $21.89 / 100,000$ (2). According to the cancer control program of the World Health Organization, the incidence and mortality rate of gastric cancer in China are $>2$ times of the global mean values (3). Presently, common treatments for gastric cancer include surgery, chemotherapy and radiation therapy. However, none of these methods have resulted in a satisfactory decrease of morbidity or mortality rates since diagnosis is usually made once the disease has reached an advanced stage (4). Therefore, it is necessary to find novel treatment approaches, including biological therapies, to treat advanced gastric cancers. Understanding the molecular basis of this disease is critical for developing novel strategies for the prevention and treatment of gastric cancer.

It is well established that cancer stem cells share multiple characteristics with embryonic stem cells (ESCs), including self-renewal and differentiation potential (5). Previous progression in stem tumor cell research has revealed that tumorigenesis may be associated with stem cells (6). Nanog homeobox (NANOG) is a transcription factor that serves a vital function in maintaining the pluripotency and self-renewal capacity of ESCs $(7,8)$. NANOG is also highly expressed in certain somatic tumors, including breast (9), prostate (10) and cervical cancers (11), thus indicating that NANOG is of vital importance in tumor transformation and progression. In addition to NANOG1, an authentic gene that encodes NANOG, there are 10 pseudogenes for NANOG in the human genome (12). NANOGP8, one of these pseudogenes, is expressed together with NANOG in multiple tumor tissues and cell lines (13). As such, NANOG/NANOGP8 may be a key component in tumor malignancy. However, the underlying molecular mechanism of NANOG/NANOGP8 during tumorigenesis remains unclear. The processes governing genesis and development of tumors are complicated, and it is therefore 
critical to identify downstream targets of NANOG/NANOGP8 in order to discover effective treatment methods. Deleted in Breast Cancer 1 (DBC1) is a nuclear protein encoded by a gene initially cloned from chromosome 8p21 that is homozygously deleted in breast cancer (14). Indeed, DBC1 mRNA is lost in several breast, lung and colon cancer cell lines (14). This has led to the suggestion that $\mathrm{DBC} 1$ is responsible for suppressing tumor development $(14,15)$. However, other studies have demonstrated that $\mathrm{DBC} 1$ is overexpressed in breast, gastric and other types of cancer, and this over expression was associated with poor prognosis (16-19). In addition, the downregulation of DBC1 has been demonstrated to inhibit gastric cancer cell proliferation and invasiveness (20). Given these conflicting findings, the function of $\mathrm{DBC} 1$ in tumorigenesis remains unclear, however it is clear that DBC1 expression serves a key function in the development and/or progression of many types of human cancer.

The underlying molecular mechanisms of NANOG/NANOGP8- or DBC1-mediated effects on gastric cancer are not well known. In the present study, NANOG and $\mathrm{DBC} 1$ expression were evaluated in gastric cancer cell lines and surgical biopsies. The effects of NANOGP8 and DBC1 suppression or upregulation on cell proliferation and apoptosis were investigated using the MKN-45 cell line. DNA microarrays and dual-luciferase assays were performed to examine the association between NANOGP8 and DBC1. The present findings indicated that NANOGP8 promoted gastric cancer progression by blinding to $\mathrm{DBC} 1$ promote region and upregulating DBC1 expression. Thus, NANOGP8 and DBC1 may serve as potential therapeutic targets for human gastric cancer.

\section{Materials and methods}

Cell culture. Human teratocarcinoma cells (N-tera), the human gastric carcinoma cell lines MKN-45, SGC-7901, HGC-27, NCI-N87, MGC803 and BGC823, as well as the human normal gastric epithelial cell line GES-1 were obtained from the Type Culture Collection of the Chinese Academy of Sciences (Shanghai, China). Cells were cultured in RPMI-1640 medium (HyClone; GE Healthcare, Logan, UT, USA) containing $10 \%$ fetal bovine serum (Gibco; Thermo Fisher Scientific, Inc., Waltham, MA, USA), under standard conditions at $37^{\circ} \mathrm{C}$ in a humidified atmosphere containing $5 \% \mathrm{CO}_{2}$.

Human tissue samples. Tissue samples were collected from 25 patients ( 10 male patients and 15 female patients, median, 60 years of age; range, 45-78 years of age) with gastric cancer who underwent tumor resection from May 2014 to May 2015 in the Affiliated Hospital of Xuzhou Medical University (Xuzhou, China). These samples were frozen in liquid nitrogen. None of the patients had been treated with chemotherapy or radiotherapy prior to surgery. Written informed consent was obtained from each patient and The Ethics Committee of Xuzhou Medical University affiliated with the Hospital approved the present study, in accordance with the Declaration of Helsinki.

Animals. A total of 10 male nude mice aged 4-6 weeks (weight, $18-20 \mathrm{~g}$ ) were used in the present study. The nude mice were obtained from Shanghai Laboratory Animal Research Center (Shanghai, China). The mice were kept in a $12 \mathrm{~h}$ light/12 h dark cycle and were provided standard lab chow and tap water ad libitum. The mice were housed at approximately $22-25^{\circ} \mathrm{C}$ with a humidity of $\sim 40-70 \%$. The nude mice were sacrificed by cervical dislocation on the 30th day subsequent to inoculation (weight, 24-26 g) following an intraperitoneal injection of $2.5 \%$ sodium pentobarbital with $0.2 \mathrm{ml} / 100 \mathrm{~g}$. All experiments were carried out in accordance with the guidelines established by the Institutional Animal Care and approved by the Ethics Committee of Xuzhou Medical University (Xuzhou, China).

Lentivirus package and stable cell construction. NANOG shRNA, negative control shRNA (21), and DBC1shRNA (22) were generated by Invitrogen (Thermo Fisher Scientific, Inc.) using sequences outlined in Table I. The concentration of shRNA was adjusted to $100 \mathrm{nmol} / \mathrm{l}$. shRNAs were then inserted into pLB plasmid vector (Addgene, Inc., Cambridge, MA, USA). pLB lentiviral particles containing shRNA were generated by transiently transfecting $293 \mathrm{~T}$ cells. Lentivirus production, concentration, and titration were each performed according to standard procedures (23).

Lipofectamine 2000 was supplied by Invitrogen. For infection, $2 \times 10^{5} \mathrm{MKN}-45$ cells were divided into four groups and cultured in 6-well plates overnight. Cells were maintained in RPMI-1640 medium, supplemented with $10 \% \mathrm{FBS}$ (Gibco) at $37^{\circ} \mathrm{C}$ in $5 \% \mathrm{CO}_{2}$. For transduction, cell culture media was removed and cells were washed twice with PBS. Then a $5 \times 10^{7} \mathrm{IU}$ lentiviral suspension containing $8 \mu \mathrm{g} / \mathrm{ml}$ polybrene was added to each well and cells were incubated at $37^{\circ} \mathrm{C}$ for $24 \mathrm{~h}$. Subsequently, the media was replaced with RPMI-1640 medium, containing $10 \% \mathrm{FBS}$ and $5 \mu \mathrm{g} / \mathrm{ml}$ puromycine to select for cells expressing the transduced vector. After $48 \mathrm{~h}$, a flow cytometer was used to sort cells containing green fluorescent protein. These processes generated five groups: MKN-45 cells, MKN-45 cells infected with lentiviral suspension expressing NANOG shRNA-1, MKN-45 cells infected with NANOG shRNA-2, MKN-45 cells infected with negative control lentiviral suspension, and MKN-45 cells infected with DBC1 shRNA.

Expression vector construction. The complete open reading frame of NANOGP8 was amplified by polymerase chain reaction (PCR) using the following primer pair (23): 5'-CAGGCA ACTCACTTTATCC-3' and 5'-TTAGGCTCCAACCATACT C-3'.

The pcDNA3.1(+) vector (Thermo Fisher Scientific, Inc.) was digested $2 \mathrm{~h}$ with $\mathrm{KpnI}$ and XhoI (Takara Biotechnology Co., Ltd., Dalian, China) at $37^{\circ} \mathrm{C}$ and the fragments subsequent to digestion were recycled. A total of $3 \mu \mathrm{l} \mathrm{PCR}$ products and $1 \mu 1$ recycled pcDNA3.1(+) were then connected using $1 \mu 1 \mathrm{~T} 4$ DNA ligase (Takara Biotechnology Co., Ltd.) at $16^{\circ} \mathrm{C}$ for $12 \mathrm{~h}$. The E. coli DH5 $\alpha$ competent cells were placed on ice for $10 \mathrm{~min}$ following the addition of $10 \mu 1$ ligation products. Subsequently, the suspension were heat shocked in a $42^{\circ} \mathrm{C}$ water bath for $90 \mathrm{sec}$ and immediately placed on ice. The bacteria solution was used to coat LB solid medium (Beyotime Institute of Biotechnology, Haimen, China) containing kanamycin $(25 \mu \mathrm{g} / \mathrm{ml})$, which was cultured for 16-20 h. Several monoclonal positive colonies were selected the next day and transferred into $4 \mathrm{ml} \mathrm{LB}$ liquid medium containing kanamycin $(25 \mu \mathrm{g} / \mathrm{ml})$, which was placed in a $37^{\circ} \mathrm{C}$ shaker to cultivate the bacteria for $12-16 \mathrm{~h}$. 
Table I. shRNA design sequences.

\begin{tabular}{ll} 
Gene & \multicolumn{1}{c}{ Sequence (5'-3') } \\
\hline NANOG shRNA-1 & 5'-AACCCTGGAACAGTCCCTTCTATATTCAAGAGATATAGAAGGGACTGTTCCAGGTTTTTTC-3' \\
& 5'-TCGAGAAAAAACCTGGAACAGTCCCTTCTATATCTCTTGAATATAGAAGGGACTGTTCCAG \\
& GGTT-3' \\
NANOG shRNA-2 & 5'-AACGGGTTAAGCTGTAACATACTTTTCAAGAGAAAGTATGTTACAGCTTAACCCTTTTTTC-3' \\
& 5'-TCGAGAAAAAAGGGTTAAGCTGTAACATACTTTCTCTTGAAAAGTATGTTACAGCTTAACC \\
& CGTT-3' \\
Control shRNA & 5'-AACTTCTCCGAACGTGTCACGTTTCAAGAGAACGTGACACGTTCGGAGAATTTTTC-3' \\
& 5'-TCGAGAAAAAATTCTCCGAACGTGTCACGTTCTCTTGAAACGTGACACGTTCGGAGAAGTT-3' \\
DBC1 shRNA & 5'-AACCCCATCTGTGACTTCCTAGAATTCAAGAGATTCTAGGAAGTCACAGATGGGTTTTC-3' \\
& 5'-TCGAGAAAAAACCCATCTGTGACTTCCTAGAATCTCTTGAATTCTAGGAAGTCACAGATG \\
& GGTT-3'
\end{tabular}

NANOG, nanoghomeobox; DBC1, deleted in breast cancer 1; sh, short hairpin.

The recombinant plasmid was extracted by E.Z.N.A. Plasmid Minikit (Omega Bio-Tek, Inc., Norcross, GA, USA), according to manufacturer's protocols, and identified by electrophoresis following digestion. The digested products were subsequently sent to Invitrogen for sequencing identification. The functional constructs were transfected using Lipofectamine 2000 (Invitrogen) into MKN-45-shDBC1 cells, which were screened using $500 \mathrm{mg} / \mathrm{l} \mathrm{G} 418$ (Gibco) for 3 weeks. This yielded MKN-45-shDBC1+NANOGP8 cells indicating stable downregulation of $\mathrm{DBC} 1$ and upregulation of NANOGP8.

Reverse transcription (RT)-PCR and sequencing of NANOG. Total RNA was extracted from MKN-45 cells and gastric cancer biopsy samples using TRIzol ${ }^{\circledR}$ reagent (Life Technologies; Shanghai, China) according to the manufacturer's protocol. Primers for NANOG, DBC1, and $\beta$-actin were as follows: NANOG forward, 5'-CAGAAGGCC TCAGCACCTAC-3' and reverse, 5'-ATTGTTCCAGGTCTG GTTGC-3'; DBC1 forward, 5'-ATGTCCCAGTTTAAGCGC CAG-3' and reverse, 5'-CAACCCCAAAGTAGTCATGCA A-3'; $\beta$-actin forward, 5'-ACTGTGCCCATCTACGAGG-3' and reverse, 5'-GAAAGGGTGTAACGCAACTA-3'. PCR was performed with the following thermocycling conditions: $94^{\circ} \mathrm{C}$ for $5 \mathrm{~min}, 94^{\circ} \mathrm{C}$ for $30 \mathrm{sec}, 53^{\circ} \mathrm{C}$ for $30 \mathrm{sec}, 72^{\circ} \mathrm{C}$ for $35 \mathrm{sec}$ for 35 cycles, with a final extension step at $72^{\circ} \mathrm{C}$ for $2 \mathrm{~min}$. Products were analyzed by electrophoresis on a $2 \%$ agarose gel. PCR products were subsequently cloned into the pCR-Blunt vector (Invitrogen) and sequenced.

Western blot analysis. MKN-45 cells were washed twice for 2 min with PBS and resuspended in radioimmunoprecipitation assay buffer (Nanjing KeyGen Biotech Co., Ltd., Nanjing, China) at $4^{\circ} \mathrm{C}$. The protein content was quantified using a bicinchoninic acid protein assay kit (Beyotime Institute of Biotechnology), according to manufacturer's protocols. A total of $200 \mu \mathrm{l}$ protein lysate was separated using 10\% SDS-PAGE and then transferred to polyvinylidene difluoride (PVDF) membranes (Nanjing KeyGen Biotech Co., Ltd.), which were incubated for $1 \mathrm{~h}$ in TBST (TBS with $1 \%$ Tween-20) containing 5\% BSA (Gibco) at room temperature. Tween-20 is a surfactant also known as polyethylene glycol sorbitan monolaurate. Membranes were subsequently incubated with primary antibodies overnight at $4^{\circ} \mathrm{C}$ as follows: Anti-NANOG (dilution, 1:5,000; cat. no. ab109250), anti-DBC1 (dilution, 1:10,000; cat. no. ab128890) and anti- $\beta$-actin (dilution, 1:1,500; cat. no. ab8226; all Abcam, Cambridge, UK) at $4^{\circ} \mathrm{C}$ overnight. Membranes were subsequently washed $5 \mathrm{~min}$ in triplicate with TBST at room temperature, incubated with horseradish peroxidase-conjugated goat anti-rabbit secondary antibody (dilution, 1:3,000; cat. no. k2034; Nanjing KeyGen Biotech Co., Ltd.) at $37^{\circ} \mathrm{C}$ for $1 \mathrm{~h}$, and washed in triplicate with TBST for $5 \mathrm{~min}$ at $37^{\circ} \mathrm{C}$. The A (Lumino) and B (Hydrogen peroxide) solutions of the electrochemiluminescence detection kit (Bio-rad, Franklin Lakes, NJ, USA) were mixed in 1:1, according to the manufacturer's protocols. The mixture was added to a PVDF membrane and allowed to react at room temperature for $5 \mathrm{~min}$ in the dark. The protein expression levels were subsequently detected through X-ray film (Kodak, Rochester, NY, USA). The bands were obtained with Imagequant LAS 4000 mini software (GE Healthcare Bio-Sciences, Pittsburgh, PA, USA) and quantified with Quantity One 4.62 software (Bio-rad).

Cell proliferation assay. Following cell transfection with sh-NANOGP8, the effect of NANOGP8 silencing on cell proliferation was measured using an MTT assay according to the manufacturer's protocol. Control and transfected cells were seeded at a density of $5 \times 10^{3}$ cells/well in a 96 -well flat-bottom plate and cultured for $6 \mathrm{~h}$ at $37^{\circ} \mathrm{C}$. MTT reagent (20 $\mu 1,5 \mathrm{mg} / \mathrm{ml})$ was then added to each well, and cells were further incubated at $37^{\circ} \mathrm{C}$ for $4 \mathrm{~h}$. Absorbance at $490 \mathrm{~nm}$ was measured using a microplate reader at 0, 24, 48 and $72 \mathrm{~h}$. Each experiment was performed in triplicate and repeated three times. The proliferation rate was calculated using the following formula: Proliferation rate $=$ survival rate $=\left[\left(\mathrm{OD}_{\text {test }}-\mathrm{OD}_{\text {negative }}\right.\right.$ control $) / O D_{\text {negative control }}$ x $100 \%$.

Flow cytometry analysis. Annexin V-APC (Allophycocyanin; BD Biosciences, Franklin Lakes, NJ, USA) apoptosis detection was used in accordance with manufacturer instructions to analyze apoptosis rate. Cells were dissociated using trypsin 
then centrifuged at $100 \mathrm{x} \mathrm{g}$ for $5 \mathrm{~min}$ at room temperature. Cells were subsequently washed twice for 2 min with PBS at room temperature and resuspended at a density of $5 \times 10^{5}$ cells/mlin binding buffer(BD Biosciences). Subsequently, $5 \mu \mathrm{l}$ Annexin V-APC and $5 \mu 1$ 7AAD (7-Aminoactinomycin D) were added to the cell suspension, which was then incubated at room temperature and protected from light for $15 \mathrm{~min}$. Data was acquired using the FACS Calibur Flow Cytometer (BD Biosciences) and results were analyzed using FlowJo software V10 platform (Tree Star Inc., Ashland, OR, USA). Each experiment was repeated three times.

Colony forming assay. Cells were harvested and plated at a density of 800 cells/well in six-well plates. Following incubation for two weeks at $37^{\circ} \mathrm{C}$, cells were washed twice with PBS and fixed with $100 \%$ methyl alcohol for 15 min at room temperature. Methyl alcohol was then removed by washing the wells twice for 2 min with PBS and the cells were stained with Giemsadye for $20 \mathrm{~min}$ at room temperature and flushed with double distilled water. Clone formation was quantified under phase contrast microscopy (magnification, $\mathrm{x} 400$ ). A clone was defined as containing more than 50 cells. Each assay was performed in triplicate.

Tumorigenecity assay in nude mice. Cells were harvested and resuspended in PBS at a density $1 \times 10^{7}$ cells $/ \mathrm{ml}$. Six-week old male athymic nude mice were subcutaneously injected in the right armpit region with $0.2 \mathrm{ml}$ cell suspension. Two groups of mice were injected with MKN-45-NC and MKN-45-shNANOGP8 stable cells ( $\mathrm{n}=5 /$ group) respectively. Tumor size was measured using calipers every 5 days. Tumor volume was calculated using the formula $\left(\mathrm{LxW}^{2}\right) / 2$, where $\mathrm{L}$ is the length and $\mathrm{W}$ is the width of the tumor. At 30 days after injection, mice were sacrificed and tumor volume and weight were measured.

DNA microarray analysis. DNA microarray analysis was performed by Kangchen BioTechCo., Ltd. (Shanghai, China). NANOG silenced and negative control MKN-45 cells were analyzed by Nimble Gen Human Gene Expression Microarrays, which were comprised of 29,250 genes. The total RNA was extracted from cells, reverse transcribed into cDNA, and marked with $\mathrm{Cy} 3$ dyes and Nimble Gen Microarray hybridization. Microarrays were washed with buffer solution I for $5 \mathrm{~min}$ at room temperature and with buffer solution II for $60 \mathrm{sec}$ at $37^{\circ} \mathrm{C}$ (Roche Diagnostics Indianapolis, IN, USA). Subsequently to being washed in an ozone-free environment, the slides were scanned using the Axon Genepix4000B microarray scanner (Molecular Devices, LLC, Sunnyvale, CA, USA). The data was collected using NimbleScan software V2.5 (Roche Diagnostics, Basel, Switzerland). The results were normalized and input into Agilent GeneSpringGX 11.0 software (Agilent Technologies, Inc., Santa Clara, CA, USA) for further analysis. The selection standard for differential gene expression was a ratio $\geq 0.5$.

Rescue experiment. Total RNA was extracted from sh-control, sh-NANOG, sh-DBC1 and sh-DBC1+NANOG cells. RT-quantitative PCR (RT-qPCR) was performed with the PrimerScript RT reagent kit (Takara Biotechnology Co., Ltd., Dalian, China) and SYBR-Green real-time PCR Master Mix

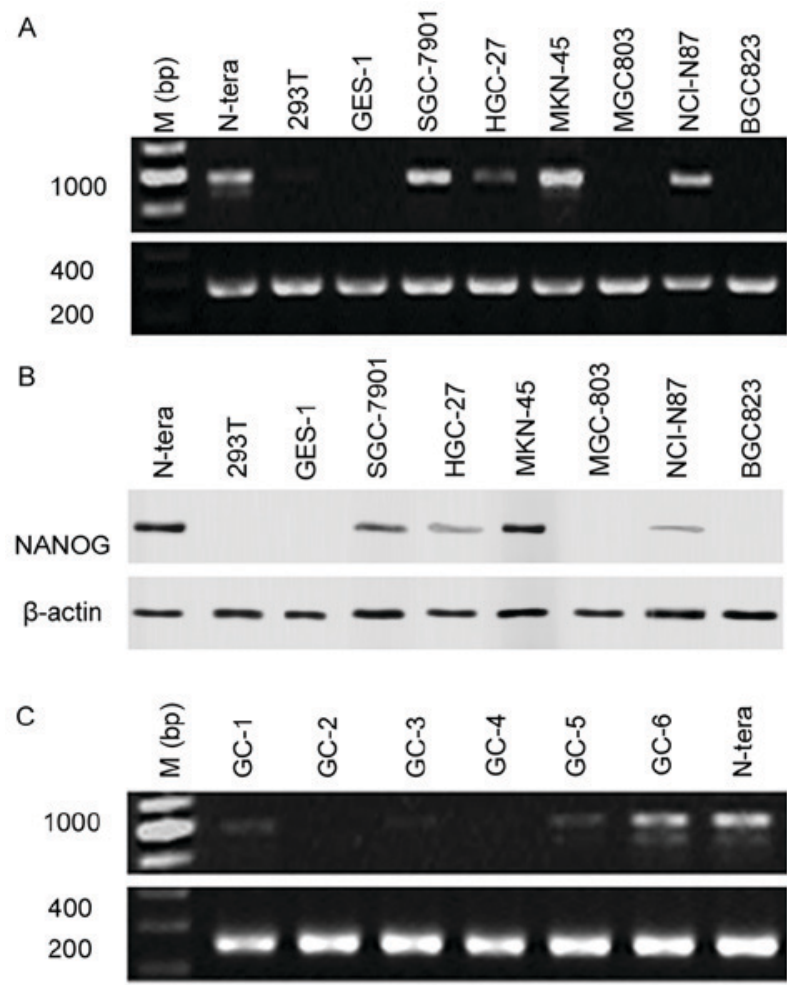

Figure 1. NANOG mRNA and protein expression in gastric cancer cell lines and surgical specimens. (A) NANOG mRNA expression levels in gastric cancer cell lines. (B) NANOG protein expression levels in gastric cancer cell lines. (C) NANOG mRNA expression levels in surgical specimens. M, marker, NANOG, nanoghomeobox.

(Takara Biotechnology Co., Ltd. Dalian, China) according to the manufacturer's protocol. The primer sequences were the same as those aforementioned. Relative quantifications were obtained via Cq values, and each sample for NANOGP8, DBC1, and $\beta$-actin was run in triplicate. The expression levels of NANOGP8 and $\mathrm{DBC} 1$ in gastric cancer cells was calculated using the $2^{-\Delta \Delta C \mathrm{r}}$ relative quantification method with $\beta$-actin as a reference (24).

Dual-luciferase assays. The predicted promoter region of DBC1 gene was from -2000 base pairs to +500 base pairs (data not shown). A DBC1 promoter luciferase reporter vector was constructed, designated pGL3-DBC1. For a dual-luciferase assay, 293T cells were cultured without antibiotics overnight and then co-transfected with pGL3-DBC1, pcDNA3.1(+)-NANOGP8, and the reference vector pRL-TK. After $24 \mathrm{~h}$, cells were lysed using PBS, and their luciferase activities measured using a Dual-Luciferase Reporter Assay System kit (Promega Corporation, Madison, WI, USA), according to the manufacturer's protocols.

Predicting NANOG transcription factor binding sites by MEME-chip software 5.0.1. Using MEME-chip 5.0.1 software (http://meme-suite.org/tools/meme-chip), three known motif features (Motif) of NANOG transcription factor binding site were identified from the transcription factor Motif database (TRANSFAC http://www.gene-regulation.com/pub/databases. $\mathrm{html}$ transfac), and their corresponding IDs were the sequences of the three Motifs of EN0298, M01247 and M01123. The open region of the chromosome contained in the promoter 
Table II. Sequence detection of NANOG expression in gastric cancer cells and surgical samples.

\begin{tabular}{lcccccccc}
\hline & \multicolumn{1}{c}{ Nucleotide } & & \\
\cline { 2 - 8 } Sample & 47 & 144 & 165 & 246 & 276 & 531 & 759 & 798 \\
\hline NANOG1 (NM_024865.2) & C & G & T & G & G & T & G & C \\
NANOGP8 (NG_004093) & A & A & T & T & G & C & C & C \\
N-tera & C & G & T & G & G & T & G & C \\
MKN-45 & A & A & T & T & G & C & C & C \\
SGC-7901 & C & A & T & T & G & C & C & C \\
HGC-27 & A & A & T & T & G & C & C & C \\
NCI-N87 & C & A & T & T & G & C & C & C \\
GC-1 & A & A & C & T & G & C & C & C \\
GC-5 & C & A & T & T & G & C & C & C \\
GC-6 & A & A & T & T & G & C & C & C \\
\hline
\end{tabular}

NANOG, nanoghomeobox.

region corresponding to the DBC1 was extracted and Motif Screening analysis was performed on the Motif sequences of EN0298, M01247 and M01123 in the open region of the chromosome.

Statistical analysis. Data were analyzed using SPSS 16.0 software (SPSS, Inc., Chicago, IL, USA). Results are depicted as the mean \pm standard deviation. A Student's t-test was used to compare means between groups. To compare values between different groups, a one-way analysis of variance was used and the least significant difference test method was used as the post-hoc test. $\mathrm{P}<0.05$ was considered to indicate a statistically significant difference.

\section{Results}

NANOG mRNA and protein expression in gastric cancer cell lines and surgical specimens. To determine NANOG mRNA and protein expression levels in gastric cancer, total RNA and protein was collected from the human gastric carcinoma cells lines BGC823, SGC7901, MKN-45, HGC-27, MGC-803 and NCI-N87, as well as from the normal human gastric epithelial cell line GES-1. NANOG mRNA and protein expression levels were examined by RT-PCR and western blotting, respectively. Data indicated that NANOG mRNA (SGC-7901, MKN-45, NCI-N87) and protein (SGC-7901, HGC-27, MKN-45, NCI-N87) expression was increased in gastric cancer cells compared with GES-1/293T normal epithelial cells, as well as MGC803/BGC823 gastric cancer cells (Fig. 1A and B), with NANOG mRNA and protein expression highest in MKN-45 cells. NANOG mRNA expression levels was also analyzed on 25 gastric cancer patient specimens using PCR, with NANOG mRNA expression identified in 6 samples (Fig. 1C).

It was confirmed using PCR that $\mathrm{N}$-tera cells expressed NANOG1 mRNA. However, the PCR products in the MKN-45, SGC-7901, HGC-27, and NCI-N87 cells, as well as in the gastric cancer surgical specimens were highly homologous with the NANOGP8 gene. This suggested that the main NANOG gene expressed by gastric cancer cell lines was NANOGP8 (Table II). The NANOG shRNA target sequence was identical between the NANOG and NANOGP8 genes, thus the NANOG shRNA may also be used to silence NANOGP8 mRNA expression.

Downregulation of NANOGP8 inhibits cell proliferation and promotes apoptosis in human gastric carcinoma cell lines. To observe the effects of NANOGP8 silencing on MKN-45 cells, cell proliferation capacity was evaluated using MTT and colony formation assays. Observation of cell proliferation for $72 \mathrm{~h}$ indicated that the proliferation rate of the sh-NANOG-1 and sh-NANOG-2 groups was significantly inhibited compared with control and parental cells (Fig. $2 \mathrm{~A} ; \mathrm{P}<0.05$ ). The effects of NANOGP8 silencing on apoptosis were then investigated using flow cytometry analysis, and it was identified that the apoptosis rate of the sh-NANOG-1 and sh-NANOG-2 groups was significantly increased compared to control or parental cells (Fig. 2B). To further evaluate proliferation ability, a colony formation assay was performed which revealed that the sh-NANOG-1 and sh-NANOG-2 groups formed smaller and fewer colonies than the parental or control cells (Fig. 2C). These results indicate that the suppression of NANOGP8 expression in human gastric cancer cells inhibits cell proliferation and promotes cell apoptosis in vitro. In addition, the effects were greater in the shNANOG-2 group than the shNANOG-1 group. Therefore, shNANOG-2 was selected for subsequent analyses.

Downregulation of NANOGP8 expression in human gastric cancer cells inhibits tumor growth. To determine whether long-term suppression of NANOGP8 in MKN-45 cells affected tumor growth in vivo, a tumorigenicity assay was performed in nude mice. Cell lines were injected subcutaneously into the right armpit region of nude mice. Tumor size and weight measurements revealed that nude mice injected with NANOGP8-silenced cells generated smaller and lighter tumors compared to sh-control cells (Figs. 3 and 4). 


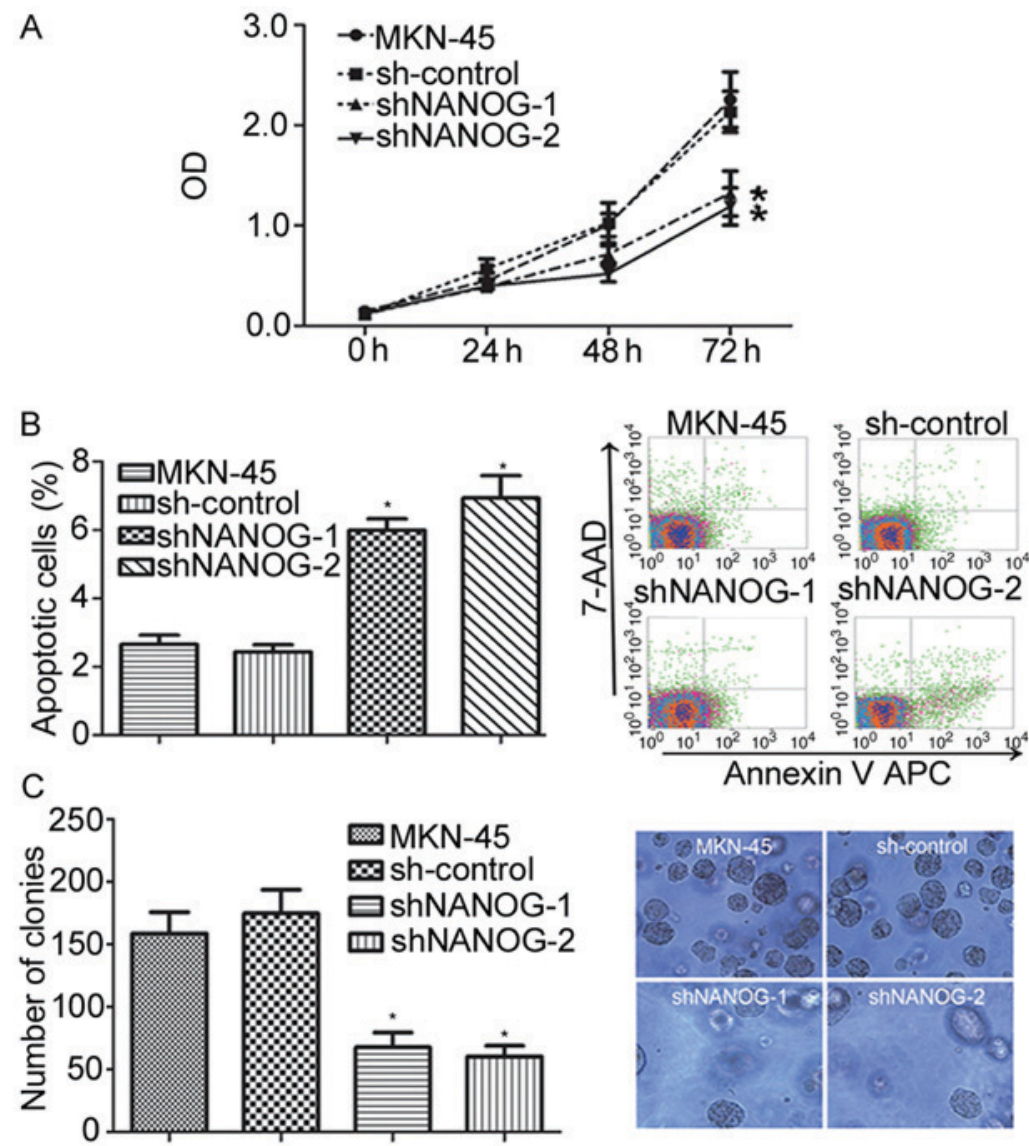

Figure 2. Downregulation of NANOGP8 expression in human gastric cancer cells. (A) The effect of NANOGP8 silencing on MKN-45 cell proliferation. (B) The effect of NANOGP8 silencing on MKN-45 cell apoptosis. (C) The effect of NANOGP8 silencing on MKN-45 proliferation (magnification, $\mathrm{x} 400$ ). "P<0.05 vs. sh-control. NANOG, nanoghomeobox; OD, optical density.

DBC1 $m R N A$ and protein expression in gastric cancer cell lines and surgical specimens. To identify downstream targets of NANOGP8, DNA microarray analysis was performed. The results suggested multiple gene expression changes following knockdown of NANOGP8 expression. In particular, DBC1 was significantly downregulated (data not shown). Previous research has demonstrated that $\mathrm{DBC} 1$ is overexpressed and associated with poor prognosis in gastric cancer (15). DBC1 also has influences on gastric cancer cell proliferation and invasiveness (19). We subsequently hypothesized that $\mathrm{DBC} 1$ was a differentially expressed gene and has an intrinsic association with the NANOGP8 gene to participate in the occurrence and development of gastric cancer. Therefore, RT-PCR and western blotting were used to detect whether DBC1 expression was altered in gastric cancer cell lines and surgical specimens. The results, as predicted, indicated that DBC1 mRNA expression was increased in gastric cancer cells compared to normal gastric epithelial cells. Furthermore, DBC1 protein expression was identified in $4 / 6$ NANOG-expressing gastric cancer surgical specimens (Fig. 5).

Rescue experiment. To further confirm the association between NANOGP8 and DBC1 expression, MKN-45 cells were divided into four groups as follows: sh-control, sh-NANOGP8, sh-DBC1 and shDBC1+NANOGP8. The results indicated that DBC1 expression was markedly downregulated following NANOGP8 knockdown. However, downregulation of DBC1 did not affect NANOG mRNA expression and the effect of

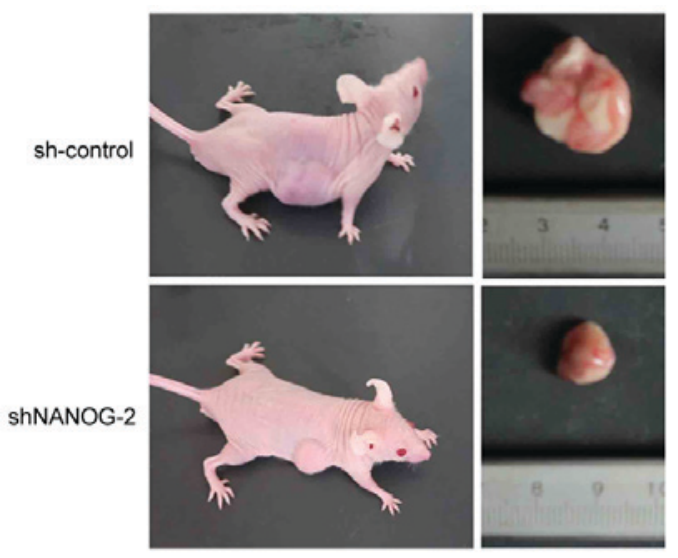

Figure 3. Tumors from nude mice injected with sh-control cells or shNANOG-2 cells.
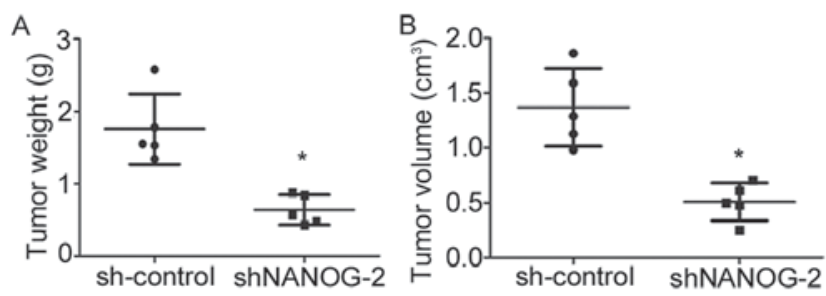

Figure 4. The effect of NAONGP8 silencing on tumor growth in nude mice. (A) Tumor weight. (B) Tumor volume. ${ }^{*} \mathrm{P}<0.05$ vs. sh-control. 
A
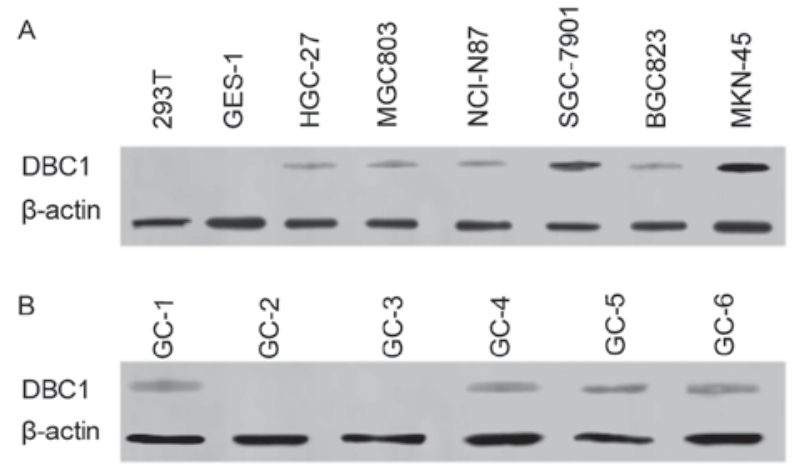

Figure 5. DBC1 mRNA and protein expression in gastric cancer cell lines and surgical specimens. (A) DBC1 mRNA expression levels in gastric cancer cell lines. (B) DBC1 protein expression levels in surgical specimens. DBC1, deleted in breast cancer 1 .

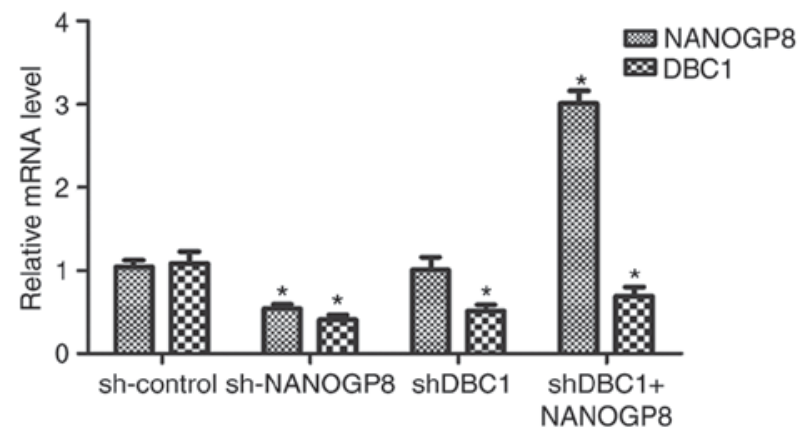

Figure 6. mRNA expression of NANOGP8 and DBC1 in sh-control, sh-NANOGP8, sh-DBC1, and shDBC1+NANOGP8 cells. "P $<0.05$ vs. sh-control. NANOG, nanoghomeobox; DBC1, deleted in breast cancer 1 .

silencing $\mathrm{DBC} 1$ on MKN-45 cells could be rescued by overexpression of NANOGP8, indicating that NANOGP8 could regulate DBC1 mRNA expression (Fig. 6).

To confirm the aforementioned results, MTT, flow cytometry and colony formation assays were performed. The results indicated that silencing DBC1 inhibited cell proliferation and promoted apoptosis relative to sh-control cells. Meanwhile, the effects of DBC1 downregulation on cell proliferation and apoptosis could be rescued by upregulation of NANOGP8 (Fig. 7). Taken together, these findings indicated that NANOGP8 promoted gastric cancer progression by regulating $\mathrm{DBC} 1$.

NANOGP8 promotes gastric cancer cell progression by transactivating $D B C 1$. The binding site for NANOGP8 within the $\mathrm{DBC} 1$ promoter region was analyzed. Based on the known motif sequence signature of NANOGP8, motif screening analysis was performed from M01123 Motif to M01247 Motif. The results revealed that the NANOGP8 binding site with $\mathrm{DBC} 1$ was in the $\mathrm{DBC1}$ promoter region (Fig. 8).

To test whether NANOG regulates DBC1 transcription, a dual-luciferase reporter assay was performed. pGL3-DBC1 or pGL3-Basic vectors were co-transfected with pcDNA3.1-NANOGP8 or pcDNA3.1 vectors into 293T cells. Cellular luciferase activity was measured at $24 \mathrm{~h}$ following transfection. The results revealed that NANOGP8 transactivated the DBC1 promoter (Fig. 9; $\mathrm{P}<0.05$ ). Taken together, these results

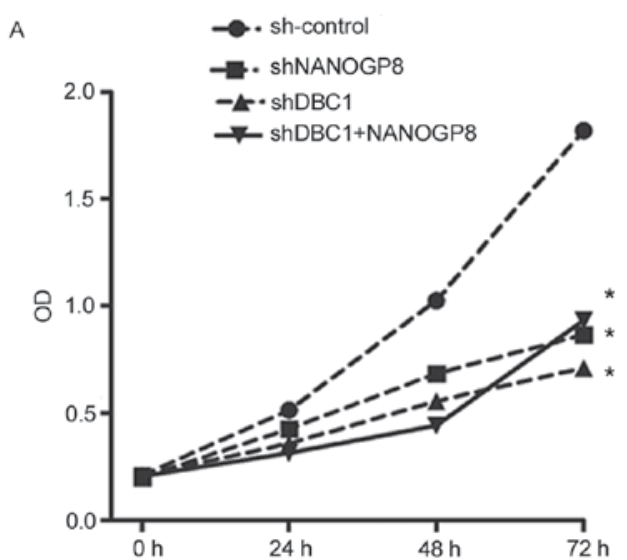

B
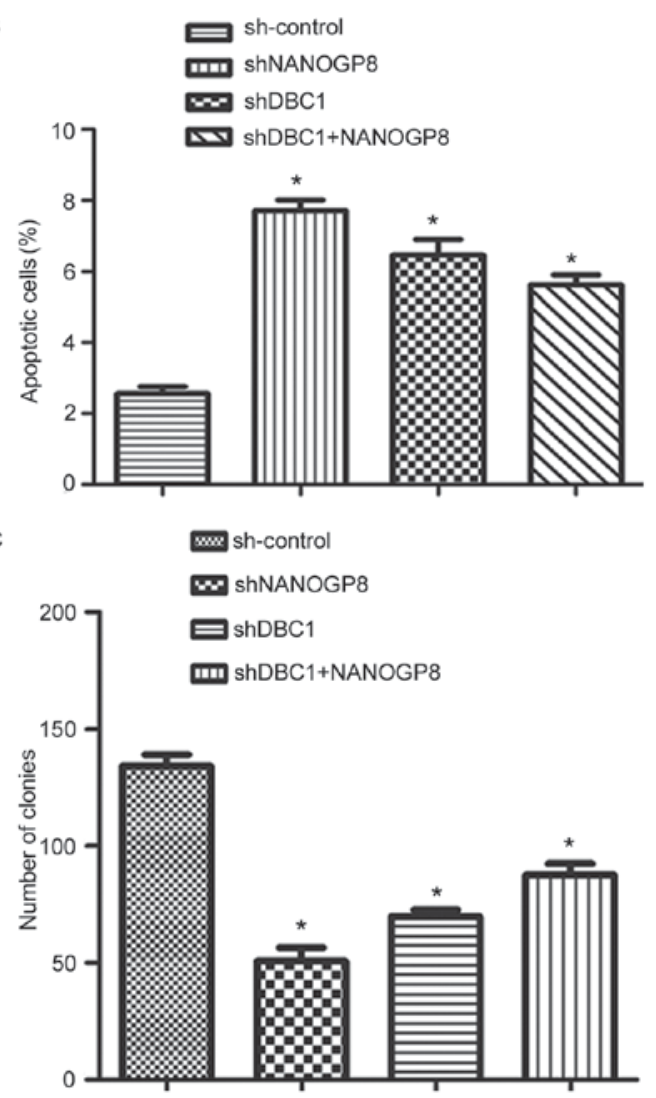

Figure 7. Downregulation of NANOGP8 or DBC1 expression in human gastric cancer cells. (A) The effect of NANOGP8 or DBC1 silencing on MKN-45 cell proliferation. (B) The effect of NANOGP8 or DBC1 silencing on MKN-45 cell apoptosis. (C) The effect of NANOGP8 or DBC1 silencing on $\mathrm{MKN}-45$ cells proliferation. ${ }^{*} \mathrm{P}<0.05$ vs. sh-control. OD, optical density; NANOG, nanoghomeobox; DBC1, deleted from breast cancer 1 .

indicated that NANOGP8 promotes gastric cancer cell progression by transactivating $\mathrm{DBC} 1$ expression.

\section{Discussion}

The objective of the present study was to determine the effects of NANOGP8 in gastric cancer cell proliferation, apoptosis and tumorigenicity, and resolve the underlying molecular mechanisms. NANOG, a transcription factor expressed in primordial germ cells and embryonic stem cells, is an important regulatory factor for maintaining gastric cancer stem cells self-renewal and pluripotency (25). In addition, NANOGP8 expression regulates 
A

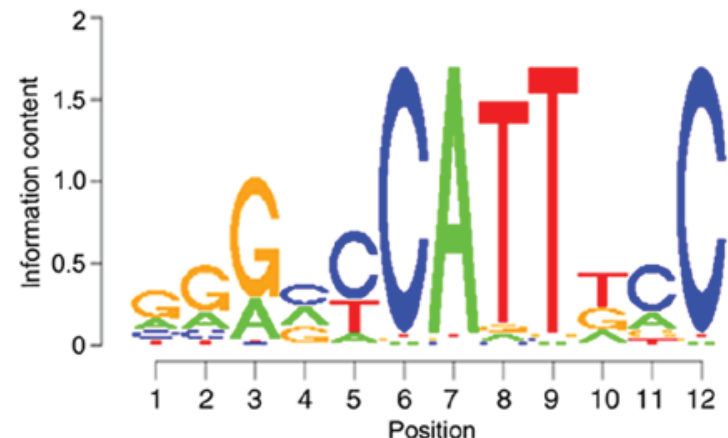

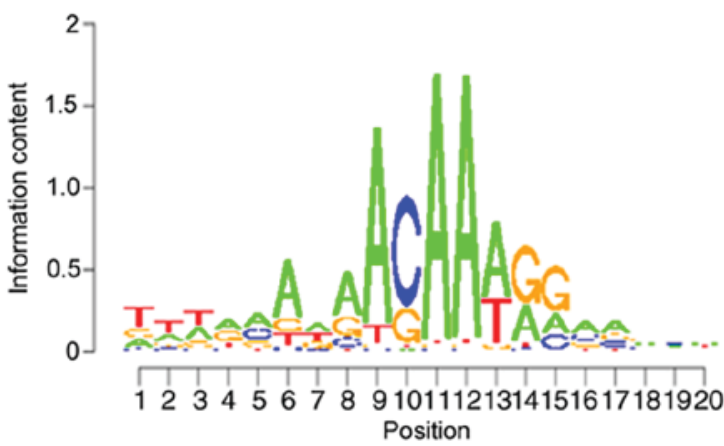

B

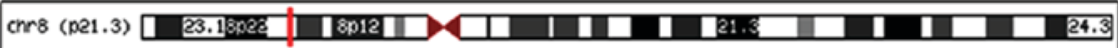

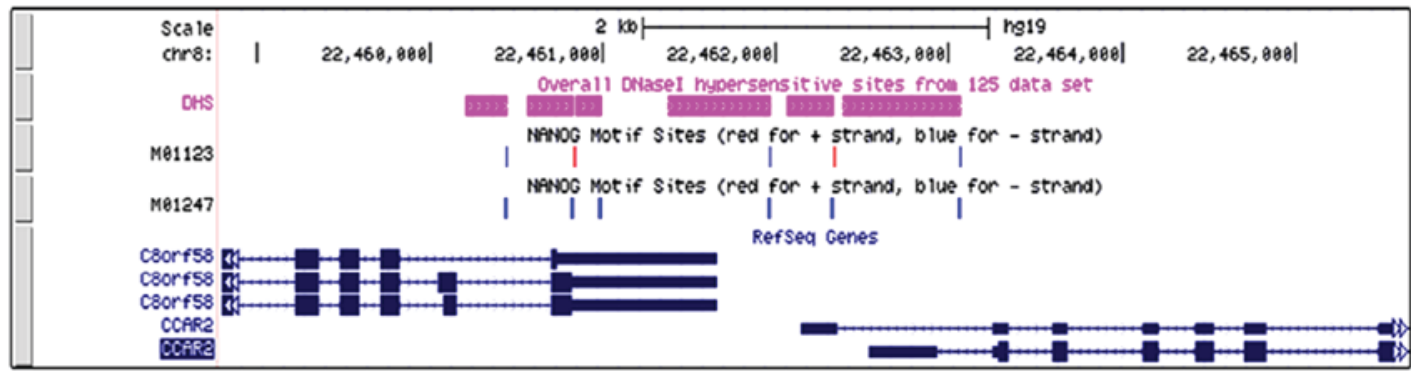

Figure 8. The NANOGP8 binding site within the DBC1promoter region. (A) Motif sequence signature of NANOGP8. (B) The predicted binding site of the NANOGP8 transcription factor. NANOG, nanoghomeobox.

proliferation and migration inhuman gastric cancer SGC-7901 cell line (26). In the present study, the differentiation status between SGC-7901 and MKN-45 cell lines differed. However, the effects of NANOGP8 on proliferation and apoptosis are consistent in SGC-7901 and MKN-45 cell lines, indicating that NANOG, serving as a promoter of gastric cancer progression, is an independent factor in gastric cancer cell differentiation status.

It has been reported that NANOGP8 overexpression significantly promotes the proliferation of tumor cells in vitro and in vivo $(27,28)$. Chiou et al $(29)$ also demonstrated that NANOG is positively associated with late stage progression and poorer prognosis for patients with oral cancer. However, the underlying NANOGP8-mediated mechanisms of tumor development remain unknown. In the present study, NANOG was overexpressed in most gastric cancer cell lines, and NANOG mRNA expression was detected in $6 / 25$ gastric surgical specimens. These results demonstrate that NANOG, as a cell-fate regulatory molecule known to be important for ESC self-renewal, may serve a novel function in gastric cancer progression. NANOG1 is an authentic gene that encodes NANOG. NANOG1 and NANOGP8 genes responsible for NANOG, encode similar polypeptides that differ from NANOG1 by only six nucleotides and two amino acids (30), thus their gene functions are similar and products are almost indistinguishable due to high degree of homology between them. The results of sequencing indicated that the products of gastric cancer cells and specimens were highly homologous with regard to the NANOGP8 gene. Therefore, it was hypothesized that NANOGP8 is likely a primary contributor of NANOG protein expression in gastric cancer.

The in vitro study results suggested that downregulating NANOGP8 expression inhibited cell proliferation, colony formation and promoted cell apoptosis in MKN-45 cells. An

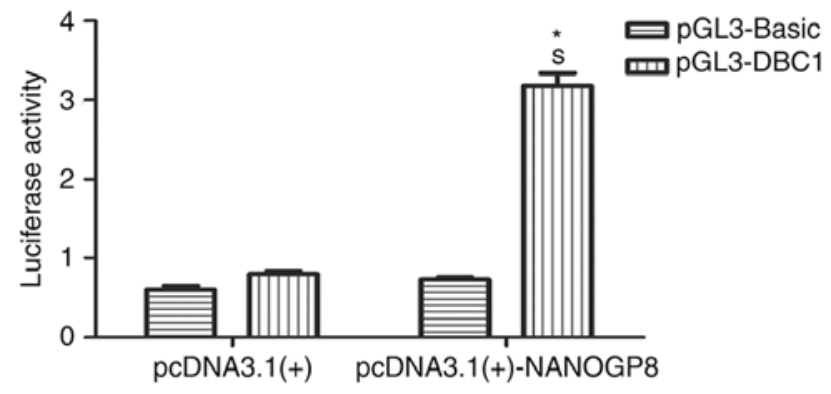

Figure 9. Luciferase assay detection of $\mathrm{DBC} 1$ promoter activation by NANOGP8. ${ }^{*} \mathrm{P}<0.05$ vs. pGL3-Basic; ${ }^{\mathrm{S}} \mathrm{P}<0.05$ vs. pcDNA3.1(+) co-transfected with pGL3-DBC1. DBC1, deleted from breast cancer 1 .

in vivo tumorigenicity assay in nude mice was also employed to verify this assumption. The gathered data indicated that NANOGP8 acted as an oncogene in gastric carcinomas. Furthermore, DBC1 has previously been associated with human breast cancer (31) and, in the present study, identified as a target gene of NANOG8. However, rather than being deleted, DBC1 expression is increased incertain humancancers (16-19). Silencing DBC1 in MKN-45 cells could be rescued by overexpression of NANOGP8. Furthermore, NANOGP8 protein was identified to directly bind to the promoter region of DBC1. It was therefore hypothesized that NANOGP8 may modulate tumor growth and metastasis through directly regulating the expression of DBC1.

To conclude, the results of the present study demonstrated that NANOGP8 serves as an oncogene to promote proliferation and suppress apoptosis in MKN-45 human gastric cancer cells. The role of NANOGP8 gene was observed through transcriptional regulation by binding to the $\mathrm{DBC} 1$ promoter region. Further study is required in order to examine whether 
the NANOGP8/DBC1 pathway is associated with clinicopathological characteristics of gastric cancer.

\section{Acknowledgments}

Not applicable.

\section{Funding}

The present study was supported by National Natural Science Foundation of China (grant no. 81470303), The Jiangsu Province Natural Science Foundation of China (grant no. BK20141140) and natural science research projects in the universities in Jiangsu province (grant no. 14KJB320021).

\section{Availability of data and materials}

The datasets used and/or analyzed during the current study are available from the corresponding author on reasonable request.

\section{Authors' contributions}

LL, GJ and JZ designed the experiment. LL, SF and JC performed the experiment. RF and QZ analyzed the data. LL, RF and JZ wrote the manuscript. All authors read and approved the final manuscript.

\section{Ethics approval and consent to participate}

All patients were required to provide written informed consent prior to their inclusion in the present study. The study was approved by the Ethical Committee of The Affiliated Hospital of Xuzhou Medical University.

\section{Patient consent for publication}

All patients provided written informed consent for the publication of their data.

\section{Competing interests}

The authors declare that they have no competing interests.

\section{References}

1. Ferlay J, Soerjomataram I, Dikshit R, Eser S, Mathers C, Rebelo M, Parkin DM, Forman D and Bray F: Cancer incidenceand mortality worldwide: Sources, methods and majorpatterns in GLOBOCAN 2012. Int J Cancer 136: E359-E386, 2015.

2. Chen W, Zheng R, Zhang S, Zhao P, Zeng H, Zou X and He J: Annual report on status of cancer in China, 2010. Chin J Cancer Res 26: 48-58, 2014

3. Ferlay J, Shine HR, Bray F, Forman D, Mathers C and Parkin DM: Estimates of worldwide burden of cancer in 2008: GLOBOCAN 2008. Int J Cancer 127: 2893-2917, 2010.

4. Wadhwa R, Taketa T, Sudo K, Blum MA and Ajani JA: Modern oncological approaches to gastric adenocarcinoma. Gastroenterol Clin North Am 42: 359-369, 2013.

5. Colmont CS, Harding KG, Piguet V and Patel GK: Human skin cancer stem cells: A tale of mice and men. Exp Dermatol 21: 576-580, 2012.

6. Reya T, morrison SJ, Clarke MF and Weissman IL: Stem cells, cancer, and cancer stem cells. Nature 414: 105-111, 2001.

7. Chambers I, Colby D, Robertson M, Nichols J, Lee S, Tweedie S and Smith A: Functional expression cloning of Nanog, a pluripotency sustaining factor in embryonic stem cells. Cell 113: 643-655, 2003.
8. Mitsui K, Tokuzawa Y, Itoh H, Segawa K, Murakami M, Takahashi K, Maruyama M, Maeda M and Yamanaka S: The homeoprotein Nanog is required for maintenance of pluripotency in mouse epiblast and ES cells. Cell 113: 631-642, 2003.

9. Ben-Porath I, Thomson MW, Carey VJ, Ge R, Bell GW, Regev A and Weinberg RA: An embryonic stem cell-like gene expression signature in poorly differentiated aggressive human tumors. Nat Genet 40: 499-507, 2008.

10. Gu G, Yuan J, Wills M and Kasper S: Prostate cancer cells with stem cell characteristics reconstitute the original human tumor in vivo. Cancer Res 67: 4807-4815, 2007.

11. Ye F, Zhou C, Cheng Q, Shen J and Chen H: Stem-cell-abundant proteins Nanog, Nucleostemin and musashil are highly expressed in malignant cervical epithelial cells. BMC Cancer 8: 108, 2008.

12. Fairbanks DJ and Maughan PJ: Evolution of the NANOG pseudogene family in the human and chimpanzee genomes. BMCEvol Biol 6: 12, 2006.

13. Zhang J, Wang X, Chen B, Suo G, Zhao Y, Duan Z and Dai J: Expression of Nanog gene promotes NIH3T3 cell proliferation. Biochem Biophys Res Commun 338: 1098-1102, 2005.

14. Hamaguchi M, Meth JL, von Klitzing C, Wei W, Esposito D, Rodgers L, Walsh T, Welcsh P, King MC and Wigler MH: DBC2, A candidate for a tumor suppressor gene involved in breast cancer. Proc Natl Acad Sci USA 99: 13647-13652, 2002.

15. Kim JE, Chen J and Lou Z: p30 DBC is a potential regulator of tumorigenesis. Cell Cycle 8: 2932-2935, 2009.

16. Cha EJ, Noh SJ, Kwon KS, Kim CY, Park BH, Park HS, Lee H, Chung MJ, Kang MJ, Lee DG, et al: Expression of DBC1 and SIRT1 is associated with poor prognosis of gastric carcinoma. Clin Cancer Res 15: 4453-4459, 2009.

17. Hiraike H, Wada-Hiraike O, Nakagawa S, Koyama S, Miyamoto Y, Sone K, Tanikawa M, Tsuruga T, Nagasaka K, Matsumoto Y, et al: Identification of DBC1 as a transcriptional repressor for BRCA1. Br J Cancer 102: 1061-1067, 2010.

18. Kang Y, Jung WY, Lee H, Lee E, Kim A and Kim BH: Expression of SIRT1 and DBC1 in Gastric Adenocarcinoma. Korean J Pathol 46: 523-531, 2012.

19. Zhang Y, Gu Y, Sha S, Kong X, Zhu H, Xu B, Li Y and Wu K: $\mathrm{DBC} 1$ is over-expressed and associated with poor prognosis in colorectal cancer. Int J Clin Oncol 19: 106-112, 2014.

20. Bae JS, Park SH, Kim KM, Kwon KS, Kim CY, Lee HK, Park BH, Park HS, Lee H, Moon WS, et al: CK2 $\alpha$ phosphorylates $\mathrm{DBC} 1$ and is involved in the progression of gastric carcinoma and predicts poor survival of gastric carcinoma patients. Int J Cancer 136: 797-809, 2015.

21. Cao J, Meng FJ, Li L, Lu C, Zhou J, Cheng H, Chen W, Chen C and Xu KL: Expression of NANOG gene in acute lymphoblastic leukemia cells and construction of lentiviral vector carrying NANOG specific shRNA. Zhongguo Shi Yan Xue Ye Xue Za Zhi 22: 275-279, 2014 (In Chinese).

22. Qin B, Minter-Dykhouse K, Yu J, Zhang J, Liu T, Zhang H, Lee S, Kim J, Wang L and Lou Z: DBC1 functions as a tumor suppressor by regulating p53 stability. Cell Rep 10: 1324-1334, 2015.

23. Cao J, Chen C, Zeng L, Li L, Li Z and Xu K: Engineered regulatory $T$ cells prevent graft-versus-host disease while sparing the graft versus-leukemia effect after bone marrow transplantation. Leuk Res 34: 1374-1382, 2010.

24. Livak KJ and Schmittgen TD: Analysis of relative gene expression data using real-time quantitative PCR and the 2(-Delta Delta C(T)) method. Methods 25: 402-408, 2001.

25. Takaishi S, Okumura T and Wang TC: Gastric cancer stem cells. J Clin Oncol 26: 2876-2882, 2008.

26. Jiang Z, Liu Y and Wang C: Oncogenic NANOGP8 expression regulates cell proliferation and migration through the Akt/mTOR signaling pathway in human gastric cancerSGC-7901cell line. Onco Targets Ther 9: 4859-4866, 2016.

27. Zhang J, Wang X,Li M,Han J, Chen B, Wang B and Dai J: NANOGP8 is a retrogene expressed in cancers. FEBS J 273: 1723-1730, 2006.

28. Uchino K, Hirano G, Hirahashi M, Isobe T, Shirakawa T, Kusaba H, Baba E, Tsuneyoshi M and Akashi K: Human Nanog pseudogene 8 promotes the proliferation of gastrointestinal cancer cells. Exp Cell Res 318: 1799-1807, 2012.

29. Chiou SH, Yu CC, Huang CY, Lin SC, Liu CJ, Tsai TH, Chou SH, Chien CS, Ku HH and Lo JF: Positive correlations of Oct-4 and NANOG in oral cancer stem-like cells and high-grade oral squamous cell carcinoma. Clin Cancer Res 14: 4085-4095, 2008.

30. Booth HA and Holland PW: Eleven daughters of NANOG. Genomics 84: 229-238, 2004

31. Zannini L, Buscemi G, Kim JE, Fontanella E and Delia D: DBC1 phosphorylation by ATM/ATR inhibits SIRT1 deacetylase in response to DNA damage. J Mol Cell Biol 4: 294-303, 2012. 